\title{
Centaurium Erythraea Extracts Exert Vascular Effects through Endothelium- and Fibroblast-dependent Pathways
}

Authors

Alae Chda ${ }^{1,2}$, Mohamed El Kabbaoui ${ }^{1}$, Paula Fresco ${ }^{2}$, Dany Silva ${ }^{2}$, Jorge Gonçalves ${ }^{2,3}$, Andreia P. Oliveira ${ }^{4}$, Paula B. Andrade ${ }^{4}$, Patrícia Valentão ${ }^{4}$, Abdelali Tazi ${ }^{1}$, Kaouakib El Abida ${ }^{1}$, Rachid Bencheikh ${ }^{1}$

Affiliations

1 Laboratory Bioactives Molecules, Faculty of Sciences and Techniques, University Sidi Mohamed Ben Abdellah, Fes, Morocco

2 Laboratory of Pharmacology, Department of Drug Sciences, Faculty of Pharmacy, University of Porto, Porto, Portugal

$3 I_{3} S$ Institute for Research and Innovation in Health Sciences, University of Porto, Porto, Portugal

4 REQUIMTE/LAQV, Laboratory of Pharmacognosy, Department of Chemical Sciences, Faculty of Pharmacy, University of Porto, Porto, Portugal

Key words

Centaurium erythraea, Gentianaceae, hypertension, endothelium-dependent relaxation, cholinomimetic effects, $\mathrm{K}^{+}$channel mediated hyperpolarization, fibroblast proliferation, phenolic composition

received May 28, 2019

revised September 30, 2019

accepted October 3, 2019

Bibliography

DOI https://doi.org/10.1055/a-1023-8918

Published online October 23, 2019 | Planta Med 2020; 86:

121-131 @ Georg Thieme Verlag KG Stuttgart · New York I ISSN 0032-0943

Correspondence

Prof. Dr. Jorge Gonçalves

Laboratory of Pharmacology, Department of Drug Sciences, Faculty of Pharmacy, University of Porto

Rua de Jorge Viterbo Ferreira 228, 4050-313 Porto, Portugal Phone: + 351220428611

jgoncalves@ff.up.pt
$\Theta$ Supporting information available online at http://www.thieme-connect.de/products

\section{ABSTRACT}

Centaurium erythraea is a plant used in traditional medicine for several cardiovascular disorders, namely hypertension, but there is no scientific evidence able to provide a molecular basis for its claimed antihypertensive effects. After a preliminary screen of extracts obtained from sequential extraction of C. erythraea aerial parts, effects of the methanolic fraction (MFCE) on changes in perfusion pressure of isolated rat mesenteric vascular bed (MVB) and in rat cardiac fibroblasts proliferation were investigated, gathering information on the mechanisms involved in endothelium-dependent effects and their dependence on a pro-proliferative stimulus. The HPLC-DAD determination of the phenolics content of MFCE revealed the presence of 22 phenolic compounds. MFCE reduced $(63.3 \pm 3.9 \% ; n=4)$ perfusion pressure in MVB and almost completely abrogated the Ang II-induced increase in cardiac fibroblasts proliferation. Reduction of the perfusion pressure caused by MFCE was endothelium-dependent and occurred in parallel with an increase in NO release. These effects were inhibited by muscarinic receptor antagonists, by L-NAME (a NO synthase inhibitor), and by ODQ (a soluble guanylate cyclase inhibitor). Experiments revealed that effects required the involvement of $\mathrm{K}^{+}$channels, being inhibited by tetraethylamonium (TEA; a $\mathrm{Ca}^{2+}$ activated $\mathrm{K}^{+}$channels inhibitor) and by glibenclamide (an ATP-sensitive $\mathrm{K}^{+}$channels inhibitor). In conclusion, extracts from C. erythraea, particularly the compounds present in the MFCE, induce endothelium-dependent vasodilation and prevent fibroblast proliferation induced by angiotensin II, which can account for the claimed antihypertensive effects of $C$. erythraea in traditional medicine.

\section{Introduction}

Centaurium erythraea Rafn. (C. erythraea), commonly known as "centaury," is a shrub species, belonging to the Gentianaceae family. This native plant is widely distributed in the Mediterranean region, where it is one of the most widely used in traditional medicine. According to several ethnopharmacologic surveys, C. ery- thraea is used for the treatment of digestive, [1] renal, hepatic, respiratory, and rheumatic diseases [2]. It is also taken as an antidiabetic agent [3] and to treat cardiovascular diseases, namely hypertension [3-6].

In vitro and in vivo studies support some of these traditional uses: extracts of $C$. erythraea were shown to possess antispasmodic [7], antioxidant [8], diuretic [9], gastroprotective [10], and anti- 


\begin{tabular}{|c|c|}
\hline \multicolumn{2}{|c|}{ ABBREVIATIONS } \\
\hline AqFCE & $\begin{array}{l}\text { aqueous fraction from sequential extraction } \\
\text { of } C \text {. erythraea aerial parts }\end{array}$ \\
\hline DAF & diaminofluorescein-FM diacetate \\
\hline DFCE & $\begin{array}{l}\text { dichloromethane fraction from sequential } \\
\text { extraction of } C \text {. erythraea aerial parts DPPH•2,2- } \\
\text { diphenyl-1-picrylhydrazyl radical }\end{array}$ \\
\hline EAFCE & $\begin{array}{l}\text { ethyl acetate fraction from sequential extraction } \\
\text { of } C \text {. erythraea aerial parts }\end{array}$ \\
\hline EDHF & endothelium derived hyperpolarizing factor \\
\hline EDRF & endothelium-derived relaxing factors \\
\hline FRAP & ferric reducing antioxidant power \\
\hline HFCE & $\begin{array}{l}\text { hexane fraction from sequential extraction } \\
\text { of } C \text {. erythraea aerial parts }\end{array}$ \\
\hline L-NAME & $\mathrm{N}$-nitro-L-arginine methyl ester \\
\hline MFCE & $\begin{array}{l}\text { methanolic fraction from sequential extraction } \\
\text { of } C \text {. erythraea aerial parts }\end{array}$ \\
\hline MVB & rat mesenteric vascular bed \\
\hline ODQ & $1 \mathrm{H}$-[1,2,4]oxadiazolo[4,3-a]quinoxalin-1-one \\
\hline SNP & sodium nitoprusside \\
\hline TEA & tetraethylamonium \\
\hline
\end{tabular}

diabetic properties [11]. Recently, it has also been shown that $C$. erythraea methanolic extract protects red blood cells from oxidative damage in diabetic rats [12].

In spite of the relevance of the use of extracts of $C$. erythraea in hypertension, the molecular mechanisms of its antihypertensive effects remain to be elucidated. Its clarification is crucial not only to understand the effectiveness of the consumption of these extracts but also to understand the risks associated with their use. The present study aimed at improving the knowledge about the mechanism of action of $C$. erythraea extracts in the vascular system, proceeding a line of investigation that demonstrated smooth muscle relaxant effects of the aqueous extract in the isolated rabbit jejunum [7]. Therefore, after a more general screening measuring the scavenging activity and vasorelaxation caused by different extracts obtained from the sequential extraction of $C$. erythraea aerial parts, the most active one (methanolic extract) was studied with more detail. Its composition was characterized and its effects on endothelium-dependent relaxation (a mechanism of short-term control of vascular function) and on fibroblast proliferation (a mechanism of medium- and long-term control of cardiac and vascular wall remodeling) were investigated.

\section{Results}

In order to identify which extract had a more pronounced scavenging activity, the hexane fraction (HFCE), the dichloromethane fraction (DFCE), the ethyl acetate fraction (EAFCE), the methanolic fraction (MFCE), and the aqueous fraction (AqFCE) from sequential extraction of $C$. erythraea aerial parts were tested using the DPPH.radical scavenging assay. Ascorbic acid (0.1-10 mg/ $\mathrm{mL}$ ) was used as positive control which presented an $\mathrm{IC}_{50}$ value of $0.11 \mathrm{mg} / \mathrm{mL}(0.10-0.12 \mathrm{mg} / \mathrm{mL} ; \mathrm{n}=4)$

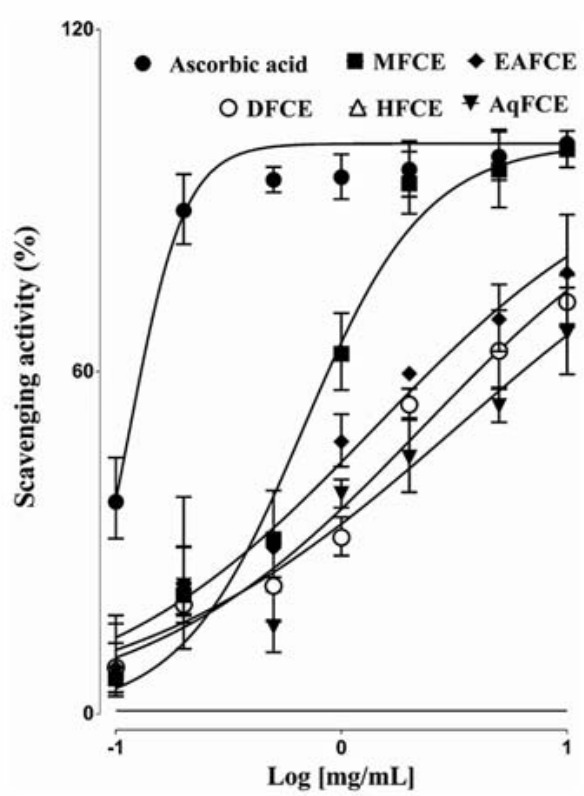

- Fig. $1 \mathrm{DPPH}^{*}$ radical-scavenging activity of the extracts obtained by sequential extraction of $C$. erythraea aerial parts: MFCE, EAFCE, DFCE, HFCE, and AqFCE. Ascorbic acid was used as positive control. Absorbance values were converted to scavenging effects (\%) considering $100 \%$ the maximal effect of ascorbic acid. Data plotted are means of replicate scavenging effect values (\%) \pm SEM of 4 independent experiments. For the sake of simplicity, symbols indicating significant values are not included in the figure. However, the effects of ascorbic acid were significant $(p<0.05)$ for all concentrations tested whereas the effects of MFCE, EAFCE, DFCE, and AqFCE reached significance for concentrations $\geq 0.5 \mathrm{mg} / \mathrm{mL}$.

With the exception of HFCE, all extracts showed scavenging activity, which was concentration-dependent. Within the concentration range tested, MFCE was able to reach a maximum of scavenging activity identical to that caused by ascorbic acid ( $\bullet$ Fig. 1). MFCE was also shown to be the most potent: the $I C_{50}$ values of MFCE were (mg/mL): $0.67(0.60-0.76)$ followed by EAFCE, DFCE, and AqFCE [1.39 (1.22-1.58), 2.25 (1.96-2.59), and 3.2 (2.583.96)], respectively $(n=4)$.

An augmented production of oxygen free radicals can determine endothelium dysfunction and diminish its ability to induce relaxation of vascular smooth muscle cells [9]. To investigate whether the extracts that presented radical scavenging activity also influenced vascular reactivity, they were tested in doses ranging from 0.03 to $3.0 \mathrm{mg}$, on MVB preparations with preserved endothelium (confirmed by administration of a bolus of $5.5 \mathrm{nmol}$ acetylcholine; see Methods), precontracted with phenylephrine $(10-20 \mu \mathrm{M})$ to induce a plateau value of perfusion pressure of $80-100 \mathrm{mmHg}$. A reduction of perfusion pressure below this plateau value caused by the experimental treatments was assumed to reflect a vasorelaxation.

Only the DFCE failed to decrease perfusion pressure (PP; - Fig. 2B). In terms of the potency to reduce perfusion pressure, the MFCE caused the most pronounced effect $(63.3 \pm 3.9 \%$; $n=4)$. The highest decrease in perfusion pressure caused by MFCE was 


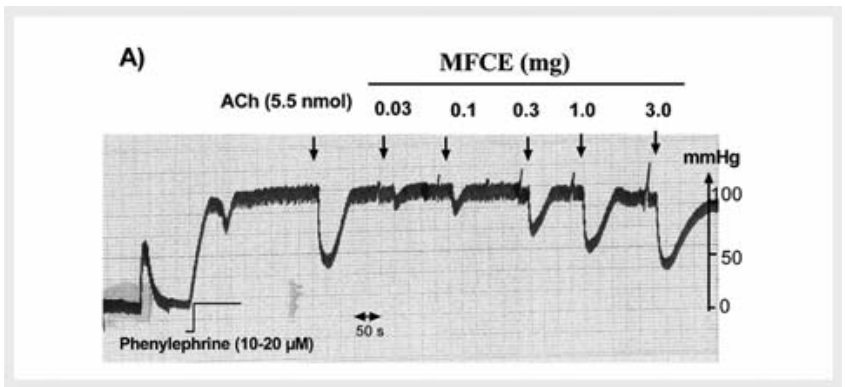

B)

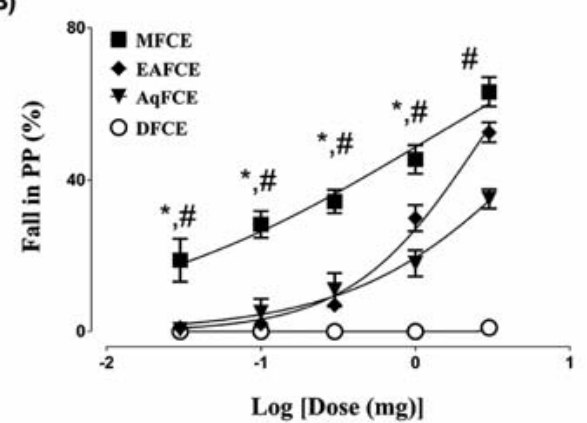

- Fig. 2 Effects of extracts obtained from sequential extraction of C. erythraea aerial parts on perfusion pressure (PP; $\mathrm{mmHg}$ ) of rat MVB precontracted with phenylephrine (10-20 $\mu \mathrm{M})$. (A) Typical trace record showing the effect of MFCE and (B) dose-response curves to DFCE, EAFCE, MFCE, and AqFCE extracts. Shown are mean \pm SEM $\left(n=4\right.$, independent experiments). ${ }^{*} p<0.05$ MFCE vs. EAFCE, ${ }^{\#} \mathrm{p}<0.05$ MFCE vs. AqFCE; 2 -way ANOVA test, followed by post hoc multiple comparisons Bonferroni's test.

followed by that caused by EAFCE $(52.7 \pm 2.6 \% ; n=4)$ and by the AqFCE $(35.0 \pm 4.0 \% ; n=4)$. Fig. $2 \mathrm{~A}$ show the typical trace of MFCE, the fraction that caused the most pronounced effect.

Since the methanolic extract (MFCE) caused the most potent vasorelaxation and radical scavenging activities, it was chosen to study, in more detail, the mechanisms involved. To investigate the endothelium-dependence of the vasorelaxation, experiments were performed in endothelium-denuded preparations (obtained by perfusing MVB with distilled water for 5-6 min). Removal of endothelium was confirmed by the lack of vasorelaxation caused by the bolus of $5.5 \mathrm{nmol}$ acetylcholine, administered after reaching the plateau value of perfusion pressure induced by phenylephrine (see Methods). Integrity of smooth muscle was confirmed by evaluating the vasorelaxation induced by $10 \mathrm{nmol}$ SNP, which was not different from that caused in endothelium preserved preparations $(70.0 \pm 3.2 \%$ vs. $76.0 \pm 4.2 \%$, respectively; $n=5)$. However, the vasorelaxation caused by all doses of MFCE was lost in endothelium-denuded preparations ( $\triangleright$ Fig. 3 ).

In order to elucidate which endothelium derived factors are involved in the vasorelaxation induced by MFCE, experiments were conducted in the presence of $100 \mu \mathrm{M}$ L-NAME, an arginine analog known to inhibit NO synthase, $1 \mu \mathrm{M}$ ODQ, an inhibitor of soluble guanylate cyclase, or $10 \mu \mathrm{M}$ indomethacin, a cyclooxygenase inhibitor. As shown in > Fig. 4, pretreatment with $100 \mu \mathrm{M} \mathrm{L-NAME}$ diminished the vasorelaxation caused by MFCE from $67.8 \pm 3.0 \%$; $n=8$ (control conditions) to $29.5 \pm 4.5 \% ; n=4$ ( $<<0.05)$. Pretreat- ment with $1 \mu \mathrm{M}$ ODQ also diminished the maximal vasorelaxation of MFCE to $27.7 \pm 0.8 \%$; $=4(p<0.05)$ whereas $10 \mu \mathrm{M}$ indomethacin did not alter significantly MFCE induced vasorelaxation.

Quantification of nitrite formation, an oxidation product of the interaction of NO with the molecular oxygen and water, was used to further monitor NO synthesis. Very low levels of nitrite were detected in the perfusate obtained from MVB precontracted with phenylephrine in the absence of MFCE $(1.38 \pm 0.08 \mu \mathrm{M} ; n=6)$. Under continuous infusion with $0.3 \mathrm{mg} / \mathrm{mL}$ of MFCE, an increase of about 5-fold in nitrite levels was detected in the perfusate of MVB precontracted with phenyelephrine, reaching $7.19 \pm$ $0.13 \mu \mathrm{M}(\mathrm{n}=6 ; \mathrm{p}<0.01$, from phenylephrine alone). This increase in nitrate levels was reduced about $57 \%$ when MVB was perfused with MFCE in the presence of $100 \mu \mathrm{M}$ L-NAME: $3.09 \pm 0.23 \mu \mathrm{M}$ ( $n=6 ; p<0.01$, from MFCE alone).

Additional evidence for an increase in NO formation induced by MFCE was obtained in experiments carried out in breast cancer MCF-7 cells using DAF-FM DA (a highly sensitive and selective fluorescent NO probe). Representative images of cells submitted to the different treatments are shown in SI (Fig. 2S, panel A). MFCE caused a marked increase in DAF-FM DA fluorescence. This increase in fluorescence was dependent on the concentration of MFCE used (0.3-1.0 mg/mL) and was prevented when cells were incubated with $500 \mu \mathrm{M}$ L-NAME, an inhibitor of nitric oxide synthase. The average values of a series of 4 independent experiments (total 24 measurements) are shown in SI (Fig 2S, panel B). This observation corroborates the hypothesis that MFCE induces nitric oxide production.

To investigate the involvement of $\mathrm{K}^{+}$channels in MVB vasorelaxation induced by MFCE, experiments were performed on preparations precontracted with $100 \mathrm{mM} \mathrm{K}^{+}$(high $\mathrm{K}^{+}$). High $\mathrm{K}^{+}$increased perfusion pressure to $94.0 \pm 7.4 \mathrm{mmHg}(n=4)$, a value identical to that induced by phenylephrine $(96.0 \pm 4.2 \mathrm{mmHg} ; \mathrm{n}=4)$. However, relaxation caused by MFCE on MVB preparations was much lower on preparations precontracted with high $\mathrm{K}^{+}$than on preparations precontracted with phenylephrine for all MFCE doses tested ( $\vee$ Fig. 5). The highest dose of MFCE reduced perfusion pressure only $14.2 \pm 1.9 \%(n=4)$.

To identify the type of $\mathrm{K}^{+}$channels involved on MFCE-induced vasorelaxation, TEA, $\mathrm{BaCl}_{2}$, and glibenclamide, inhibitors of $\mathrm{Ca}^{2+}$ activated $\mathrm{K}^{+}$channels, inwardly rectifying $\mathrm{K}^{+}$channels, and ATP sensitive $\mathrm{K}^{+}$channels inhibitor, respectively, were tested in endothelium-intact MVB preparations precontracted with phenylephrine.

As shown in > Fig. 6, $10 \mathrm{mM}$ TEA and $1 \mu \mathrm{M}$ glibenclamide, but not $100 \mathrm{mM} \mathrm{BaCl}_{2}$, reduced the relaxation caused by MFCE. The maximal vasorelaxation induced by $3 \mathrm{mg}$ MFCE was reduced by $10 \mathrm{mM}$ TEA and $1 \mu \mathrm{M}$ glibenclamide: from $66.9 \pm 2.5 \%(n=8)$ (solvent) to $14.5 \pm 2.5 \%(n=4)$ and to $38.6 \pm 2.0 \%(n=4)$, respectively $(p<0.05$; see $>$ Fig. 6$)$.

To examine the putative involvement of cholinergic mechanisms in the vasorelaxation caused by MFCE, the effect of the highest doses of MFCE was examined in the presence of atropine, a muscarinic receptor antagonist. As shown in $>$ Fig. 7, $1 \mu \mathrm{M}$ atropine prevented the reduction of perfusion pressure caused by $5.5 \mathrm{nmol}$ acetylcholine. It also prevented reduction in perfusion pressure caused by 1 and $3 \mathrm{mg}$ MFCE. Under the same experimen- 


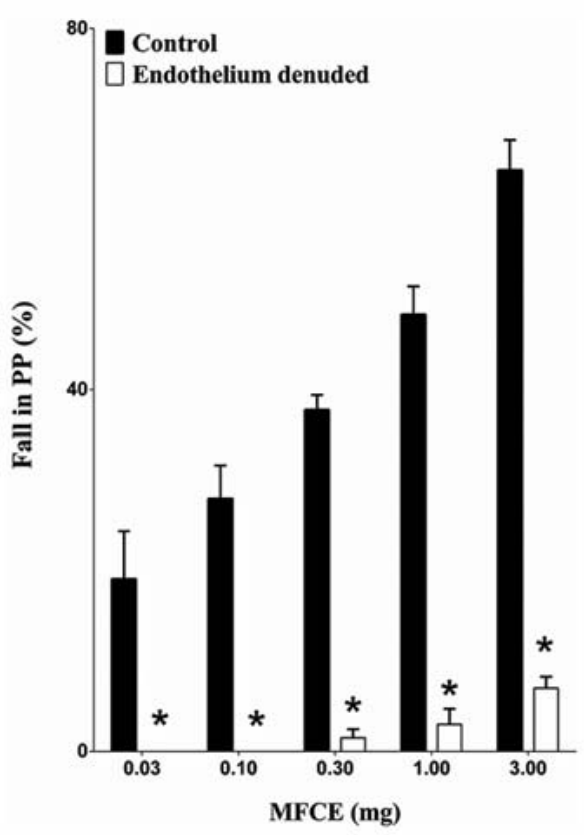

- Fig. 3 Influence of endothelium removal on the reduction of perfusion pressure (PP) of rat MVB precontracted with phenylephrine (up to $80-100 \mathrm{mmHg}$ ), caused by MFCE. Shown are mean \pm SEM $(n=5),{ }^{*} p<0.05$ from the respective control (MVB with preserved endothelium); 2-way ANOVA test, followed by post hoc multiple comparisons Bonferroni's test.

tal conditions, responses to $10 \mathrm{nmol}$ SNP (as an exogenous donor of NO) were not altered.

The involvement of muscarinic receptors in the effects of MFCE was confirmed in experiments carried out in isolated ileum, a classical model to study muscarinic responses. Carbachol (7.5 nM to $25 \mu \mathrm{M})$ was used as a selective muscarinic-receptor agonist and caused concentration-dependent contractions that were blocked by the muscarinic receptor antagonists $1 \mu \mathrm{M}$ atropine or $1 \mu \mathrm{M}$ AQ-RA 741 (Supplementary material: Fig. 1S, panel A). Similar effects to $1 \mu \mathrm{M}$ atropine or $1 \mu \mathrm{M}$ AQ-RA 741 were obtained on the contractions caused by MFCE (0.015-1.0 mg/mL) (Supporting Information, Fig. 1S, panel B).

Considering the described contribution of fibroblast proliferation to cardiovascular diseases, the effect of MFCE was tested on primary cultures of rat cardiac fibroblasts. When tested alone, MFCE $(0.01-1.0 \mathrm{mg} / \mathrm{mL}$ ) failed to alter fibroblast proliferation (3 independent experiments, data not shown). However, when cardiac fibroblasts were treated with $0.1 \mu \mathrm{M}$ angiotensin II, a known inducer of fibroblast proliferation, MFCE almost completely abrogated the Ang II-induced increase in cell proliferation ( $\triangleright$ Fig. 8).

The analysis by HPLC-DAD allowed the identification of 22 phenolic compounds in MFCE, comprising quercetin, kaempferol, and isorhamnetin derivatives, most of them being acylated with hydroxycinnamic acids ( $\triangleright$ Fig. 9 and Table 1 ). The identification of these compounds was carried out by comparing chromatographic behavior with the results recently published by our group, using

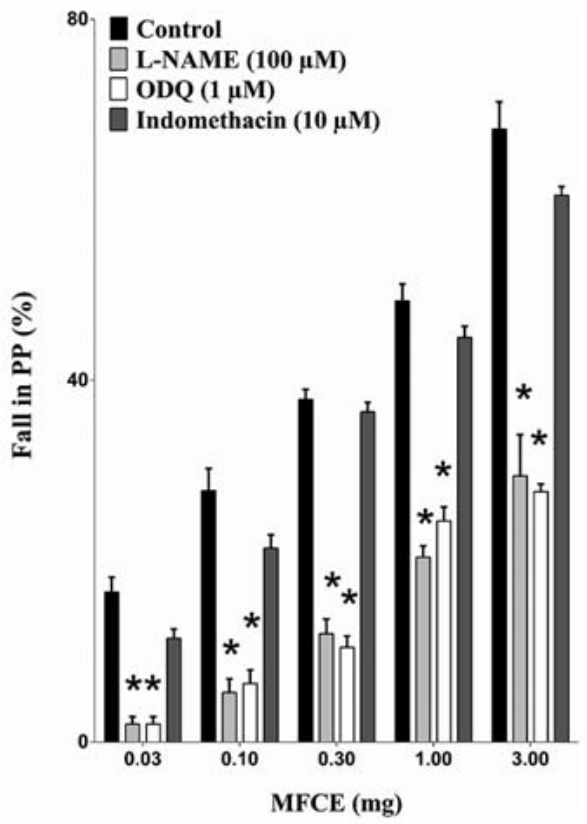

- Fig. 4 Influence of inhibition of NO and prostaglandin synthesis/ pathway on the fall of perfusion pressure (PP) of rat MVB precontracted with phenylephrine (up to $80-00 \mathrm{mmHg}$ ), caused by MFCE. MFCE $(0.03-3 \mathrm{mg})$ was tested in the absence (control) or in the presence of $100 \mu \mathrm{M}$ L-NAME, a NO synthase inhibitor, $1 \mu \mathrm{M}$ ODQ, an inhibitor of soluble guanylate cyclase, or $10 \mu \mathrm{M}$ indomethacin, a cyclooxygenase inhibitor. Shown are mean \pm SEM $(n=4-8)$; ${ }^{*} \mathrm{p}<0.05$ different from the respective control (solvent); 2-way ANOVA test, followed by post hoc multiple comparisons Bonferroni's test.

the same plant material, in which HPLC-DAD-ESI (IonTrap)/MS was used [10].

The quantification of MFCE compounds was carried out using external calibration curves resulting from 7 concentrations of the following standards: quercetin-3-O-rutinoside $\left(y=46534 \mathrm{x} ; \mathrm{R}^{2}=\right.$ 0.9996; limit of detection [LOD] $=6.10 \times 10^{-4} \mathrm{mg} / \mathrm{mL}$; limit of quantification [LOQ] $=1.85 \times 10^{-4} \mathrm{mg} / \mathrm{mL}$ ), kaempferol-3-O-rutinoside $\left(y=60151 x ; R^{2}=0.9994 ; \quad L O D=3.00 \times 10^{-4} \mathrm{mg} / \mathrm{mL} ; \quad L O Q=\right.$ $\left.9.10 \times 10^{-4} \mathrm{mg} / \mathrm{mL}\right)$ and isorhamnetin-3-O-rutinoside $(\mathrm{y}=52524 \mathrm{x}$; $\mathrm{R}^{2}=0.9995 ; \mathrm{LOD}=5.20 \times 10^{-4} \mathrm{mg} / \mathrm{mL} ; \mathrm{LOQ}=1.57 \times 10^{-3} \mathrm{mg} / \mathrm{mL}$ ). Under the chromatographic conditions described above, the calibration plots showed a good correlation. Concentrations ranging from 0.129 to $17.923 \mathrm{mg} / \mathrm{g}$ were found in this extract ( $\triangleright$ Table 1 ). Phenolic compounds were mainly represented by kaempferol derivatives (47.5\% of determined phenolic compounds), followed by quercetin heterosides (46.1\%). The most abundant compounds in these 2 groups were kaempferol-3-O-(p-coumaroyl, rhamnosyl)rutinoside-7-O-rhamnoside (14) and quercetin-3-O-(rhamnosyl)rutinoside-7-O-(p-coumaroyl)rhamnoside (11), which represent $23.3 \%$ and $18.1 \%$ of the total phenolics content, respectively. Isorhamnetin-3-O-(p-coumaroyl, rhamnosyl)rutinoside-7-O-rhamnoside (15) was the only isorhamnetin derivative identified, accounting for $6.4 \%$ of the total phenolics content ( $\bullet$ Table 1 ). 


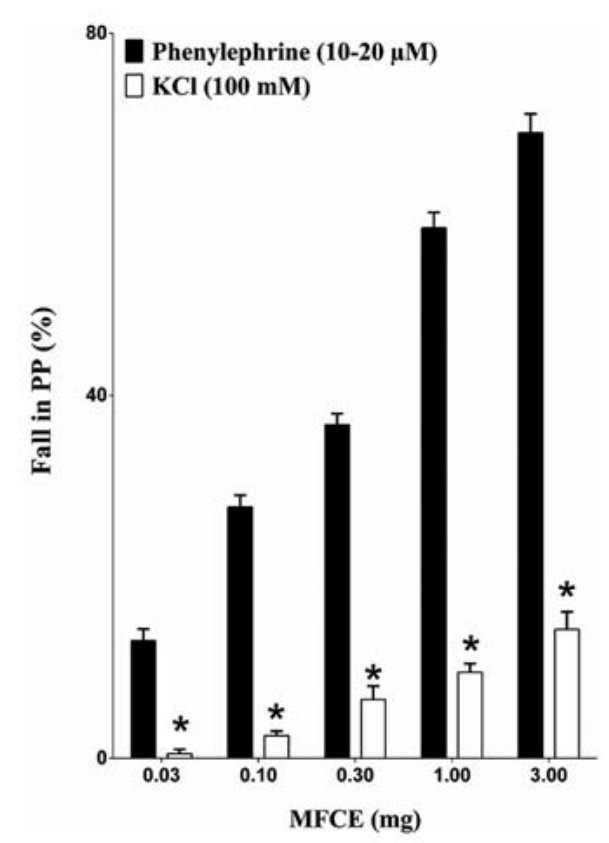

- Fig. 5 Effects of the MFCE on perfusion pressure (PP) of the rat MVB precontracted either with phenylephrine $(10-20 \mu \mathrm{M})$ or $\mathrm{K}^{+}$ $(100 \mathrm{mM})$ to identical perfusion pressure values $(80-100 \mathrm{mmHg})$. Shown are mean \pm SEM $(n=4) ;{ }^{*} p<0.05$ significantly different from the respective control (responses of MFCE on MVB preparations pre-contracted with phenylephrine); 2-way ANOVA test, followed by post hoc multiple comparisons Bonferroni's test.

\section{Discussion}

C. erythraea is used in traditional medicine to treat cardiovascular diseases, namely hypertension [3-6]. To the best of our knowledge, this is the first study that aimed to investigate the effects of $C$. erythraea extracts on the cardiovascular system. The purpose is to gather scientific evidence to better understand the real efficacy and safety of their use in traditional medicine. The main focus of the present study was to evaluate the capacity of extracts (i) to reduce oxidative stress, by measuring its radical scavenging activity; (ii) to potentiate endothelium dependent control of vascular resistance, by measuring endothelium dependent relaxation and (iii) to interfere with cardiovascular remodeling, by investigating their capacity to modulate fibroblast proliferation.

Several in vitro and epidemiological studies suggested an important role of herbal medicine extracts rich in polyphenols to preserve vascular health and to prevent cardiovascular diseases [13]. The oxidative stress results from a cellular imbalance between the effect of pro and antioxidants, in favor of the first, being considered as a factor that contributes to the atherogenic process [14]. Previous studies have shown that extracts from C. erythraea present antioxidant effects [15] using DPPH ${ }^{\circ}, 2,2$-azinobis (3-ethyl-benzothiazoline-6-sulphonic acid ammonium salt; ABTS), and/or FRAP method. In addition to in vitro data, it was showed that $C$. erythraea also prevents oxidative stress on experimentally diabetic rats [12]. The results from the present study

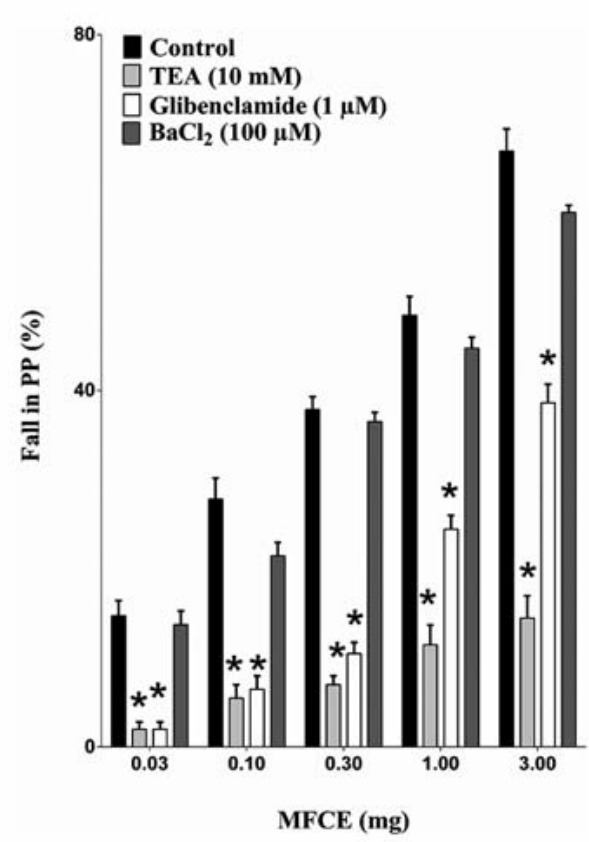

- Fig. 6 Influence of $\mathrm{K}^{+}$channel blockers (10 mM TEA; $1 \mu \mathrm{M}$ glibenclamide and $100 \mu \mathrm{M} \mathrm{BaCl}_{2}$ ) on the fall of perfusion pressure (PP) of rat MVB precontracted with phenylephrine (up to $80-100 \mathrm{mmHg}$ ) caused by the methanolic extract of $C$. eryhthraea (MFCE). Shown are mean \pm SEM $(n=4-8) ;{ }^{*} p<0.05$ significantly different from the respective control (solvent); 2-way ANOVA test, followed by post hoc multiple comparisons Bonferroni's test.

provide additional information about the nature of antioxidant effect of $C$. erythraea extracts, showing that scavenging activity was absent to moderate for the extracts with low and average polarity but important with polar methanolic extract (MFCE). The scavenging effect displayed by $C$. erythraea extracts could be a promising therapeutic agent to treat oxidative stress induced by pathological conditions.

To explore the vasoreactivity of bioactive extracts identified in the $\mathrm{DPPH}^{*}$ assay, endothelium-intact MVBs were precontracted with phenylephrine, a $\alpha_{1}$-adrenoceptor agonist. In the presence of this contracting agent, MFCE, EAFCE, and AqFCE induced dose-dependent relaxation. Interestingly, MFCE-induced vasorelaxation was more potent than that induced by EAFCE and AqFCE. Considering this difference between MFCE and other extracts, and in order to diminish the number of animals used, only the MFCE mechanism for inducement of vasorelaxant activity was studied in more detail.

The use of antioxidant therapy has been considered a valid approach to improve vascular function in case of coronary artery disease, stroke, peripheral vascular disease, hypertension, and heart failure $[16,17]$. Multiple mechanisms may be involved and mediate an improvement of the endothelium-dependent vasodilation [14] and an involvement of endothelium-dependent mechanisms on the reduction of perfusion pressure caused by MFCE extract is one of the major findings of the present study. 


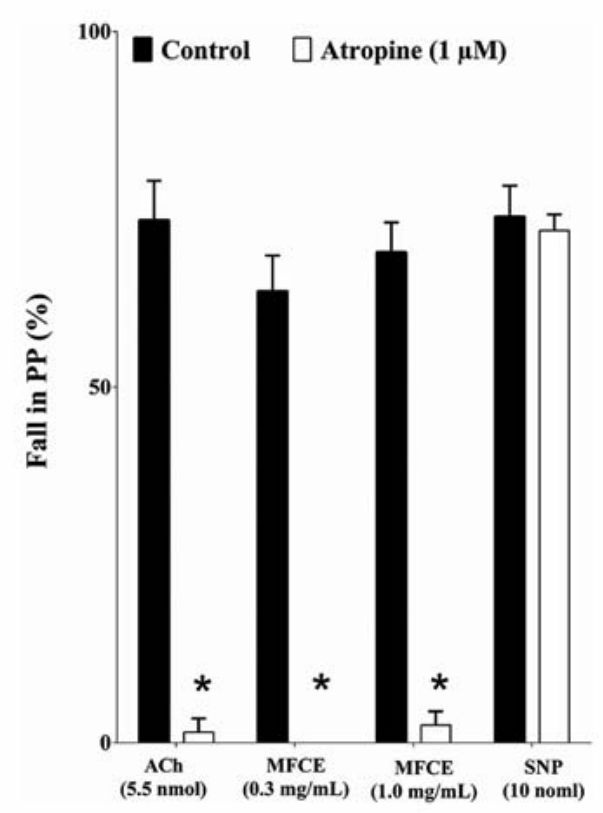

- Fig. 7 Influence of atropine $(1 \mu \mathrm{M})$ on the fall of perfusion pressure (PP) of rat MVB precontracted with phenylephrine (up to 80$100 \mathrm{mmHg}$ ) caused by the methanolic extract of $C$. eryhthraea (MFCE). Shown are mean \pm SEM $(n=4) ;{ }^{*} p<0.05$ significantly different from the respective control (absence of $1 \mu \mathrm{M}$ atropine); 2-way ANOVA test, followed by post hoc multiple comparisons Bonferroni's test.

Endothelial control of vascular tone is achieved via synthesis and/or release of a wide range of biochemical mediators, namely EDRFs and endothelium-derived contracting factors [18]. Endothelium-dependent vasodilation is believed to be achieved by 3 main EDRFs: NO, EDHF, and prostacyclin $[18,19]$. The present results support the involvement of $\mathrm{NO}$ and a probable contribution of EDHF in the vasorelaxation caused by the $C$. erythraea extract.

$\mathrm{NO}$ is a gaseous mediator that, in endothelial cells, is produced by a $\mathrm{Ca}^{2+}$-dependent constitutive isoform of NO synthase. Responses of vascular smooth muscle to NO are mainly mediated by the heme-containing soluble guanylate cyclase, causing an increase in cGMP levels, activation of protein kinase $G$, and reduction in intracellular free $\mathrm{Ca}^{2+}$ levels, leading to dilation of blood vessels [19]. The present study showed that MFCE may stimulate NO formation and the vasorelaxation be caused by activation of the NO-cGMP pathway, as it was inhibited by L-NAME, an inhibitor of NO synthase, and by ODQ, an inhibitor of guanylate cyclase. Taken together these results reveal a capacity of extracts of C. erythraea to induce NO release.

Mechanisms other than NO release may be involved in the endothelium-dependent vasodilation caused by $C$. erythraea extracts. The evidence presented in our study points to the involvement of EDHF. Release EDHF depends on activation of $\mathrm{K}^{+}$channels [19] and the results of the present study points to a $\mathrm{K}^{+}$dependence of vascular relaxation caused by MFCE: relaxation was prevented when the equilibrium potential of $\mathrm{K}^{+}$(high $\mathrm{K}^{+}$conditions) is close to the membrane potential, by reducing the electrochemical

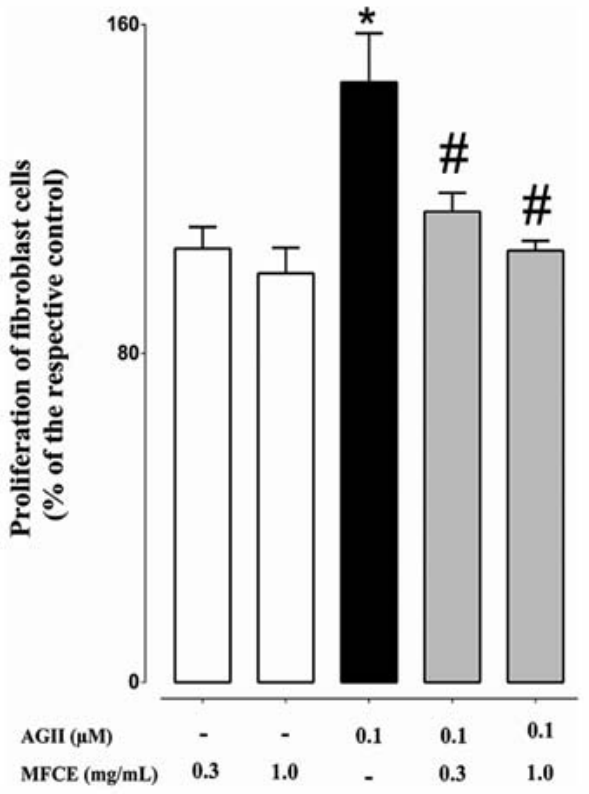

- Fig. 8 Influence of the methanolic extract of C. eryhthraea (MFCE) on proliferation of rat cardiac fibroblasts cells in the absence or in the presence of $0.1 \mu \mathrm{M}$ angiotensin II (AGII). Cells were incubated for $96 \mathrm{~h}$ with the indicated combination of drugs. Shown are mean \pm SEM of 4 independent experiments; * significantly different from solvent; \# from AGIl alone; $p<0.05$; 2-way ANOVA test, followed by post hoc Bonferroni's multiple comparisons test.

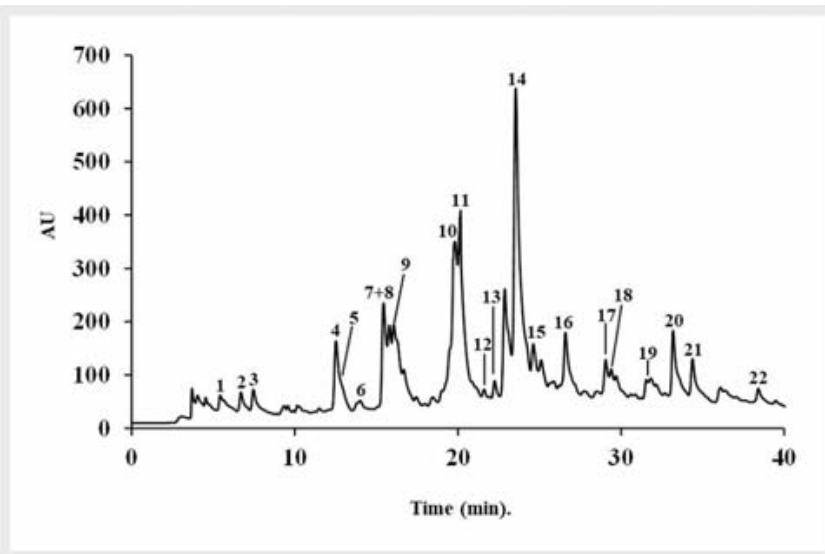

- Fig. 9 Phenolic profile of the methanolic extract obtained from the sequential extraction of $C$. erythraea aerial parts. Detection at $330 \mathrm{~nm}$. Compounds identity as in > Table 1.

gradient for $\mathrm{K}^{+}$efflux and thus preventing relaxation mediated by opening of $\mathrm{K}^{+}$channels [20]. Furthermore, relaxation seems to be mediated, at least in part, by increasing $\mathrm{K}^{+}$efflux through $\mathrm{K}_{\text {ATP }}$ and $\mathrm{K}_{\mathrm{Ca}}$ channels, according to the inhibition of this effect caused by glibenclamide (a K $\mathrm{K}_{\text {ATP }}$ channel inhibitor) and tetraethylammonium (a $\mathrm{K}_{\mathrm{Ca}}$ channel inhibitor). 
- Table 1 Phenolics content of the methanolic extract obtained from the sequential extraction of $C$. erythraea aerial parts ${ }^{\mathrm{a}}$.

\begin{tabular}{|c|c|c|c|}
\hline \multicolumn{2}{|c|}{ Compound $^{\mathrm{b}}$} & \multirow{2}{*}{$\begin{array}{c}\mathbf{m g} / \mathbf{g} \text { dry extract } \\
0.812 \pm 0.048\end{array}$} & \multirow{2}{*}{$\begin{array}{l}\% \\
1.0\end{array}$} \\
\hline 1 & Quercetin-3-O-(Rhm)Rut-7-O-Rhm & & \\
\hline 2 & Kaempferol-3-O-(Rhm)Rut-7-O-Rhm & $0.518 \pm 0.005$ & 0.7 \\
\hline 3 & Kaempferol-3-(Caff,Rhm)Rut-7-Hx & $0.146 \pm 0.006$ & 0.2 \\
\hline 4 & Quercetin-3-(Rhm)Rut-7-(Caff)Rhm & $3.091 \pm 0.042$ & 4.0 \\
\hline 5 & Quercetin-3-O-Rut-7-0-Rhm & $1.370 \pm 0.123$ & 1.8 \\
\hline 6 & Quercetin-3-(Rhm)Rut-7-(Caff)Rhm & $0.281 \pm 0.030$ & 0.4 \\
\hline $7+8$ & Kaempferol-3-O-Rut-7-O-Rhm + Kaempferol-3-(p-Coum,Rhm)Rut-7-Hx & $4.936 \pm 0.372$ & 6.4 \\
\hline 9 & Quercetin-3-(Rhm)Rut-7-(Caff)Rhm & $4.000 \pm 0.438$ & 5.2 \\
\hline 10 & Quercetin-3-(Rhm)Rut-7-(p-Coum)Rhm & $8.811 \pm 0.647$ & 11.4 \\
\hline 11 & Quercetin-3-(Rhm)Rut-7-(p-Coum)Rhm & $13.956 \pm 0.230$ & 18.1 \\
\hline 12 & Quercetin-3-(Rhm)Rut-7-(p-Coum)Rhm & $0.129 \pm 0.013$ & 0.2 \\
\hline 13 & Kaempferol-3-(p-Coum,Rhm)Rut-7-Rhm & $0.308 \pm 0.008$ & 0.4 \\
\hline 14 & Kaempferol-3-(p-Coum,Rhm)Rut-7-Rhm & $17.923 \pm 1.032$ & 23.3 \\
\hline 15 & Isorhamnetin-3-(p-Coum,Rhm)Rut-7-Rhm & $4.904 \pm 0.057$ & 6.4 \\
\hline 16 & Kaempferol-3-(p-Coum,Rhm)Rut-7-Rhm & $2.905 \pm 0.095$ & 3.8 \\
\hline 17 & Quercetin-3-(p-Coum)Rut-7-Rhm & $3.085 \pm 0.194$ & 4.0 \\
\hline 18 & Kaempferol-3-(Caff)Rut-7-Rhm & $1.669 \pm 0.140$ & 2.2 \\
\hline 19 & Kaempferol-3-(p-Coum,Rhm)Rut & $0.971 \pm 0.021$ & 1.3 \\
\hline 20 & Kaempferol-3-(p-Coum)Rut-7-Rhm & $5.433 \pm 0.052$ & 7.0 \\
\hline 21 & Kaempferol-3-(p-Coum)Rut-7-Rhm & $1.326 \pm 0.068$ & 1.7 \\
\hline 22 & Kaempfeol-3-(p-Coum)Rut-7-Rhm & $0.396 \pm 0.005$ & 0.5 \\
\hline$\Sigma$ & & $76.970 \pm 2.503$ & \\
\hline
\end{tabular}

The vasorelaxation caused by $C$. erythraea may involve activation of muscarinic receptors, which is one of the first and best known mechanisms to induce release of EDRFs [21]. The present study confirms that MFCE presents a cholinomimetic effect, and the induced vasorelaxation is secondary to activation of endothelial muscarinic receptors since it was blocked by different muscarinic receptor antagonists. The mechanisms that may be, directly or indirectly, involved in the vasodilation caused by MFCE are depicted in $>$ Fig. 10.

In addition to the well-known effect of the endothelium in the genesis of cardiovascular diseases, a role of fibroblasts is also established [22]. In hypertension, vascular changes are the result of tissue remodeling in which adventitious fibroblasts increase production of collagen, and increase wall thickness and vasoconstriction [23, 24]. These changes are also directly implicated in ventricular fibrosis [25]. Angiotensin II is a messenger that has been implicated in inducing proliferation of fibroblasts [26], and blockade of this pro-proliferative effect is assumed to be one of the mechanisms that can explain the cardiovascular therapeutic effects of angiotensin converting enzyme inhibitors $[23,26]$ and of antagonists of $\mathrm{AT}_{1}$ receptors [27]. In the present study, the MFCE extract inhibited fibroblast proliferation induced by angiotensin II, mimicking what would be expected from blockade of angiotensin II effects. Such observation suggests that, in addition to influencing endothelial function by preserving the endothelium vasodilatory function, $C$. erythraea contains compounds that may inhibit wall remodeling of the cardiovascular system, thus constituting an additional target to prevent or to treat hypertension.

Extracts have, in general, a complex composition. The qualitative profile of MFCE is identical to the one reported by Kachmar et al. [28] although quantitative differences were noticed, MFCE being richer than the aqueous extract of $C$. erythraea aerial parts ( $\triangleright$ Table 1). From the 22 flavonoid derivatives identified in MFCE, most of them were acylated with hydroxycinammic acids (3, 4, 622) ( Table 1). Quercetin, kaempferol, and isorhamnetin derivatives were also identified by comparing their UV spectra and chromatographic behavior with the results recently published by Kachmar and colleagues [10] for the aqueous extract of the same plant material using the same chromatographic conditions. With such high number of compounds present in the extract and having in mind that biological effects observed may be caused the additive or synergic effects, the attempt to identify which compounds are contributing for the observed biological effects is unfeasible. Evidence from the literature may point to relevant contributions of some compounds detected. For example, quercetin and isorhamnetin were reported to reduce oxidative stress and to improve NO bioavailability, leading to the attenuation of endothelial dysfunction [29-33], and kaempferol is known to have a re- 


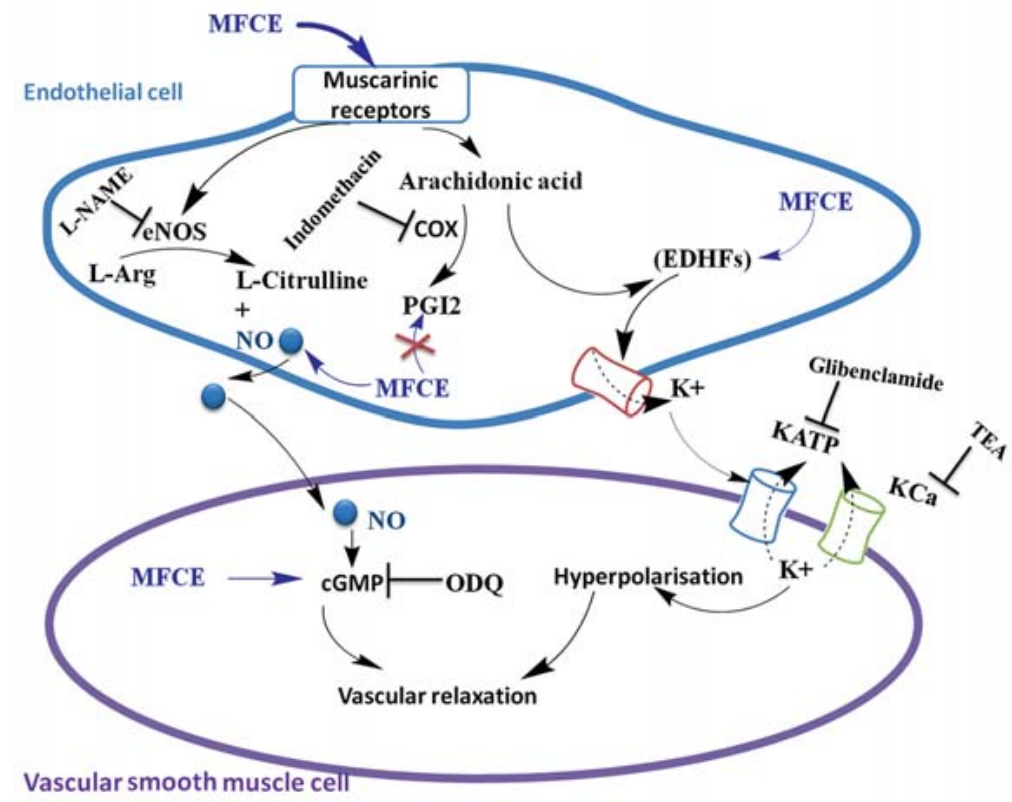

- Fig. 10 Schematic illustration proposing the targets that may be influenced directly or indirectly by the compounds present in the methanolic extract of C. eryhthraea (MFCE).

laxation effect on agonist-induced vascular contraction regardless of endothelial function [34,35]. In addition, hydroxycinnamic acids, such as p-coumaric and caffeic acids, are known to exhibit vasorelaxant and antioxidant effects [36,37].

In conclusion, $C$. erythraea extracts may alter vascular function by inducing endothelium-dependent vasodilation and preventing cardiac fibroblast proliferation. Since both effects have an impact on short and long-term vascular resistance and vessel wall remodeling, these may contribute to the claimed antihypertensive effects of $C$. erythraea in traditional medicine.

\section{Materials and Methods}

\section{Plant material}

C. erythraea was collected at north Morocco (Taounate) between May and June 2017 and stored at room temperature in a dry place prior to use. The plant was identified (Amina Bari, Department of Biology, FSDM, University Sidi Mohamed Ben Abdellah, Fez, Maroc) and registered with the specimen number MA-FSTF 14, at the Faculty of Science and Technique (Fez, Morocco). Aerial parts (leaves and flowers) were dried protected from light.

\section{Extraction}

Dried aerial parts were ground into a fine powder. Fifty grams of this powder was sequentially extracted with $300 \mathrm{~mL}$ of hexane, dichloromethane, ethyl acetate, methanol, and water, using a Soxhlet extractor. The sequential extraction yields were $2.5 \%$, $2.9 \%, 3.7 \%, 24.4 \%$, and $3.1 \%$ for HFCE, DFCE, ethyl acetate (EAFCE), methanol (MFCE), and water extracts (AqFCE), respectively. These fractions were dried and stored at $4{ }^{\circ} \mathrm{C}$, protected from light. Dry extracts were prepared in order to assure that no trace of organic solvents was detected in the highest concentration of extract used.

\section{In vitro DPPH`antioxidant assay}

The DPPH ${ }^{\bullet}$ (2,2-diphenyl-1-picryl hydrazyl radical) scavenging assay was carried out as described previously [38]. Briefly, $100 \mu \mathrm{L}$ of each dissolved extract, in concentrations of $0.1,0.2,0.5,1.0,2.0$, 5.0 , or $10 \mathrm{mg} / \mathrm{mL}$, were added to $400 \mu \mathrm{L}$ of $0.5 \mathrm{mM}$ DPPH ${ }^{\bullet}$. Thereafter, $1.5 \mathrm{~mL}$ of methanol was added and, after a 30 min incubation, absorbances were read at $517 \mathrm{~nm}$. The $\mathrm{IC}_{50}$ values (dose required to reduce $\mathrm{DPPH}^{\bullet}$ absorbance by $50 \%$ ) were calculated using nonlinear regression analysis. Ascorbic acid was used as reference antioxidant (positive control). The radical scavenging activity of extracts was estimated by calculating the absorbances ratios in the presence and in the absence of extracts and expressed as percentage of the maximal effect of ascorbic acid.

\section{Animals}

Male and female Wistar rats (250-350 g; Charles River) were used for all experiments. The animals were housed in appropriate facilities of the Institute of Biomedical Sciences of University of Porto or the Faculty of Sciences and Techniques, University Sidi Mohamed Ben Abdellah, at ambient temperature of $25 \pm 2{ }^{\circ} \mathrm{C}$. Animals had free access to food and tap water ad libitum. The present study was performed according to the guidelines of the European Communities Council Directive (86/609/EEC) and has received the Ethics Committee authorization with the reference number $\mathrm{N}^{\circ}$ 255/2018/ORBEA (ORBEA: Organismo Regulador do Bem-Estar Animal; the local organism that regulates experimental animal welfare), March 8, 2019. 


\section{Isolated MVB experiments}

The MVB was removed from Wistar rats (male or female; 300$400 \mathrm{~g}$ ) as previously described [39]. Rats were anesthetized with sodium pentobarbital $(50 \mathrm{mg} / \mathrm{kg}$, i. p.). After opening the abdominal cavity, the superior mesenteric artery was rapidly cannulated with a heparinized hypodermic needle at its origin from the abdominal aorta and immediately flushed with warm Krebs-Henseleit solution (KHS). The whole MVB was separated from the intestine by cutting close to the intestinal border and placed into a Petri dish. Once the MVB was isolated, KHS, at $37^{\circ} \mathrm{C}$, was pumped into the MVB using a peristaltic pump (Pharmacia Biotech) at a continuous constant flow of $2 \mathrm{~mL} / \mathrm{min}$. The MVB perfusion pressure was continuously measured using a pressure transducer (Capto SP844) and recorded on a universal oscillograph (508622, Harvard Apparatus Limited). The KHS had the following composition (mM): $\mathrm{NaCl} 118, \mathrm{NaHCO}_{3} 25, \mathrm{KCl} 4.7, \mathrm{MgSO}_{4} 1.2$, $\mathrm{CaCl}_{2} 2.5, \mathrm{KH}_{2} \mathrm{PO}_{4} 1.2$, and glucose 11.0 ; it was continuously oxygenated $\left(95 \% \mathrm{O}_{2}, 5 \% \mathrm{CO}_{2}\right.$; final $\left.\mathrm{pH} 7.4\right)$.

After 60 min equilibration, the preparation was constricted by infusion of phenylephrine (10-20 $\mu \mathrm{M})$, via a sidearm of the inflow of the perfusate, using a pump syringe (M365, Orion). The concentration of phenylephrine reached in the KHS was adjusted in every experiment in order to induce a stable perfusion pressure of $80-100 \mathrm{mmHg}$, as previously described [40]. An increase in perfusion pressure was interpreted as a vasoconstriction and expressed as the absolute increase in pressure $(\mathrm{mmHg})$ above the baseline and a decrease in perfusion pressure of precontracted tissue preparations interpreted as a vasodilation. Drug-induced relaxation is expressed as a percentage of the decrease in perfusion pressure, assuming $100 \%$ relaxation a return to precontraction values.

In some experiments, endothelium was removed from the $M V B$, using the following protocol: following a return to resting conditions after the initial control responses had been obtained, distilled water was included in the perfusion stream for 5-6 min to remove the endothelium from the MVB, followed by a stabilization period of $30-40 \mathrm{~min}$, as previously described $[41,42]$. The presence of functional endothelium was confirmed by the ability of a bolus of acetylcholine $(5.5 \mathrm{nmol})$ to induce more than $60 \%$ relaxation of vessels precontracted with phenylephrine. The absence of a relaxation response to acetylcholine was taken as evidence that MVBs were functionally denuded from endothelium. After a preliminary screen of each fraction, only the methanolic fraction of $C$. erythraea (MFCE) was studied in more detail. For the dose-response curve to $\operatorname{MFCE}(0.03,0.1,0.3,1.0$, and $3.0 \mathrm{mg}$ ), extract was administered by bolus injection of $10 \mu \mathrm{L}$ to $100 \mu \mathrm{L}$ into the perfusion stream close to the superior mesenteric artery canula, and its effects were measured in vessels precontracted with phenylephrine, with and without endothelium. In the experiments designed to investigate the molecular mechanisms of the vasodilation, drugs were added 20 min prior to phenylephrine, as indicated.

\section{NO determination}

The presence of NO in the MVB perfusate was determined by measuring the amount of nitrites. Nitrite determination was performed in MVB perfused with KHS containing phenylephrine, in the absence or in the presence MFCE $(0.3 \mathrm{mg} / \mathrm{mL})$, alone and in the presence of $100 \mu \mathrm{M}$ L-NAME. In order to obtain detectable nitrite levels, samples of perfusate $(6 \mathrm{~mL})$ were collected at a flow rate of $2 \mathrm{~mL} / \mathrm{min}$. Nitrites were quantified using the Griess reagent ( $1 \%$ sulfanilamide, $0.1 \%$ napthylethylenediamine, $2.5 \%$ ortophosphoric acid) [43]. Briefly, Griess reagent $(6 \mathrm{~mL})$ was mixed with an equal volume of perfusate $(6 \mathrm{~mL})$, and optical density was determined at $550 \mathrm{~nm}$, after a 10 -min incubation period. For the quantification of the nitrites present in the perfusate, a calibration curve was constructed using known concentrations $(1-50 \mu \mathrm{M})$ of sodium nitrite.

\section{Fibroblast culture and MTT cell viability assay}

Cells obtained from primary cultures of rat cardiac fibroblasts were kindly provided by Professor Paulo Correia-de-Sá (Laboratory of Pharmacology, ICBAS, University of Porto) and prepared accordingly [44], being cultured in DMEM (Sigma), supplemented with $15 \%(\mathrm{v} / \mathrm{v})$ inactivated FBS (Sigma) and $1 \%(\mathrm{v} / \mathrm{v})$ penicillinstreptomycin/amphotericin B solution. Cells were seeded in 96well plates, at a density $3 \times 10^{4}$ cells $/ \mathrm{mL}$, in a final volume $100 \mu \mathrm{L}$ of DMEM supplemented with $15 \%$ FBS and $1 \%$ penicillin-streptomycin/amphotericin B. Cells were incubated for $96 \mathrm{~h}$, at $5 \% \mathrm{CO}_{2}$ at $37^{\circ} \mathrm{C}$, in the absence or in the presence of MFCE $(0.3$ and $1 \mathrm{mg} / \mathrm{mL})$, with or without angiotensin II $(0.1 \mu \mathrm{M})$.

Cell viability was measured by a colorimetric assay with MTT, as described previously [45]. In brief, at the end of the incubation period, medium was removed and $100 \mu \mathrm{L}$ of a MTT solution in PBS $(0.5 \mathrm{mg} / \mathrm{mL})$ was added to each well, and kept in the same incubation conditions (at $37^{\circ} \mathrm{C}$ and $5 \% \mathrm{CO} 2$ ) for $4 \mathrm{~h}$, protected from the light. Thereafter, supernatants were aspired and $100 \mu \mathrm{L}$ DMSO added to each well to dissolve the formazan crystals that resulted from MTT reduction. The quantity of formazan produced (an indicator of the number of viable cells) is measured by changes in absorbance at $570 \mathrm{~nm}$, using a 96 well microplate reader (Sinergy HT, Biotek Instrumensts Inc).

\section{HPLC-DAD analysis of phenolic compounds}

The dried residue of MFCE was re-dissolved in methanol and filtered through a $0.45 \mu \mathrm{m}$ size pore membrane (Millipore). Thereafter, $20 \mu \mathrm{L}$ of re-dissolved extract was analyzed in a HPLC-DAD unit (Gilson), using a Kinetex column $(5 \mu \mathrm{m}, \mathrm{C} 18,100 \mathrm{~A}$, $150 \times 4.6 \mathrm{~mm}$; Phenomenex), following the conditions described before [10]. The mobile phase consisted of 2 phases: water-formic acid (1\%) (Phase A) and acetonitrile (Phase B), starting with $15 \% \mathrm{~B}$ and using a gradient to obtain $20 \% \mathrm{~B}$ at $15 \mathrm{~min}, 25 \% \mathrm{~B}$ at $25 \mathrm{~min}$, $30 \% \mathrm{~B}$ at $35 \mathrm{~min}$, and $60 \% \mathrm{~B}$ at $45 \mathrm{~min}$, at a flow rate of $0.5 \mathrm{~mL} /$ min. Detection was achieved with a Gilson diode array detector. Spectral data from all peaks were collected in the range of 200$400 \mathrm{~nm}$ and chromatograms were recorded at $330 \mathrm{~nm}$. The data were processed on a Clarity Software system. Peak purity was checked by the software contrast facilities. Phenolic compounds quantification was achieved by interpolation with external standard calibration curves. Since standards of all identified compounds are not commercially available, quercetin derivatives were quantified as quercetin-3-O-rutinoside, isorhamnetin derivatives as isorhamnetin-3-O-rutinoside, and kaempferol derivatives as kaempferol-3-O-rutinoside. The LOD and the LOQ were calculated 
from the residual standard deviation of the linear regression (SD), following the equations: $\mathrm{LOD}=3.3 \times \mathrm{SD}$ and $\mathrm{LOQ}=10 \times \mathrm{SD}$.

\section{Chemicals and drugs}

All solutions were prepared daily and kept on ice until used. Carbachol, acetylcholine chloride, L-NAME, phenylephrine, TEA, barium chloride $\left(\mathrm{BaCl}_{2}\right)$, angiotensin II, and atropine were dissolved in distilled water. Indomethacin was dissolved in $150 \mathrm{mM}$ $\mathrm{NaHCO}_{3}$. Glibenclamide, AQ-RA 741, and ODQ were dissolved in dimethyl sulfoxide (DMSO). The final concentration of DMSO used in the present study did not exceed $0.05 \%$, which was proven to have no effect per se on the basal tonus of the preparations or on the effects of the drugs tested.

DMEM, FBS, and L-glutamine were purchased from MerckMillipore (BioTecnómica). Quercetin-3-O-rutinoside, kaempferol3-O-rutinoside and isorhamnetin-3-O-rutinoside were from Extrasynthèse. All other drugs and solvents were purchased from Sigma-Aldrich. All reagents used in this study were of the highest available analytic grade (purity $\geq 98 \%$ ).

\section{Statistical analysis}

Statistical analysis and nonlinear regression analysis were carried out using GraphPad Prism 6.00 software. Results are presented as mean \pm standard error of the mean (SEM) of, at least, 4 independent experiments expressed as a percentage of the respective control. $\mathrm{IC}_{50}$ values were calculated using nonlinear regression analysis and presented as geometric means and 95\% confidence intervals. Comparisons between groups were performed by analysis of variance test for repeated measurements (2-way analysis of variance [ANOVA]), followed by the multiple comparison Bonferroni's t-test. Comparisons between 2 means were performed by Student's t-test. Differences were considered statistically significant at $\mathrm{p}<0.05$.

\section{Supporting Information}

Additional evidence for involvement of muscarinic receptors and NO release on the effects of MFCE was gathered by investigating the effects of MFCE on isolated rat ileum contractions and on NO release in breast cancer MCF-7 cells, using DAF-FM DA, a highly sensitive and selective fluorescent NO probe.

\section{Acknowledgements}

We are grateful to Professor Amina Bari (Department of Biology, FSDM, University sidi Mohamed Ben Abdellah, Fez, Maroc) for botanical identification, to M. Céu Pereira (Laboratory of Pharmacology, Department of Drug Sciences, Faculty of Pharmacy, University of Porto, Portugal) for technical support, and to Professor Paulo Correia-de-Sá (Laboratory of Pharmacology, ICBAS, University of Porto, Portugal) for the generous gift of fibroblast cells. AC received a scholarship from Erasmus+ International Credit Mobility - MARE NOSTRUM Consortium to support his stay, as a visiting Ph.D. student, in Porto, Portugal. This work was supported by UID/QUI/50006/2019 with funding from FCT/MCTES through national funds, by Programa de Cooperación Interreg V-A España - Portugal (POCTEP) 2014-2020 (project 0377_IBERPHENOL_6_E), and by REQUIMTE through the APO research contract (DL57/2016/CP1346/ CT0015).
Conflict of Interest

The authors declare that they have no conflict of interest.

References

[1] Merzouki A, Ed-derfoufi F, Molero Mesa J. Contribution to the knowledge of Rifian traditional medicine. II: folk medicine in Ksar Lakbir district (NW Morocco). Fitoterapia 2000; 71: 278-307

[2] Bouyahya A, Abrini J, Et-Touys A, Bakri Y, Dakka N. Indigenous knowledge of the use of medicinal plants in the north-west of Morocco and their biological activities. Eur J Integr Med 2017; 13: 9-25

[3] Bnouham M, Mekhfi H, Legssyer A, Ziyyat A. Medicinal plants used in the treatment of diabetes in Morocco. Int J Diabetes Metab 2002; 10: 33-50

[4] Bellakhdar J, Claisse R, Fleurentin J, Younos C. Repertory of standard herbal drugs in the Moroccan pharmacopoea. J Ethnopharmacol 1991; 35: $123-143$

[5] Uncini Manganelli RE, Chericoni S, Baragatti B. Ethnopharmacobotany in Tuscany: plants used as antihypertensives. Fitoterapia 2000; 71: S95S100

[6] Calvo MI, Cavero RY. Medicinal plants used for cardiovascular diseases in Navarra and their validation from official sources. J Ethnopharmacol 2014; 157: 268-273

[7] Chda A, Kabbaoui M, Chokri A, Abida K, Tazi A, Bencheikh R. Spasmolytic action of Centaurium erythraea on rabbit jejunum is through calcium channel blockade and NO release. European J Med Plants 2016; 11: 1-13

[8] Valentão P, Fernandes E, Carvalho F, Andrade PB, Seabra RM, Bastos ML. Antioxidant activity of Centaurium erythraea infusion evidenced by its superoxide radical scavenging and xanthine oxidase inhibitory activity. J Agric Food Chem 2001; 49: 3476-3479

[9] Haloui M, Louedec L, Michel JB, Lyoussi B. Experimental diuretic effects of Rosmarinus officinalis and Centaurium erythraea. J Ethnopharmacol 2000; 71: 465-472

[10] Tuluce Y, Ozkol H, Koyuncu I, Ine H. Gastroprotective effect of small centaury (Centaurium erythraea $L$ ) on aspirin-induced gastric damage in rats. Toxicol Ind Health 2011; 27: 760-768

[11] Sefi M, Fetoui H, Lachkar N, Tahraoui A, Lyoussi B, Boudawara T, Zeghal N. Centaurium erythrea (Gentianaceae) leaf extract alleviates streptozotocin-induced oxidative stress and $\beta$-cell damage in rat pancreas. J Ethnopharmacol 2011; 135: 243-250

[12] Đorđević M, Mihailović M, Arambašić Jovanović J, Grdović N, Uskoković A, Tolić A, Sinadinović M, Rajić ], Mišić D, Šiler B, Poznanović G, Vidaković M, Dinić S. Centaurium erythraea methanol extract protects red blood cells from oxidative damage in streptozotocin-induced diabetic rats. J Ethnopharmacol 2017; 202: 172-183

[13] Varadharaj S, Kelly OJ, Khayat RN, Kumar PS, Webb AJ, Miller MR. Role of dietary antioxidants in the preservation of vascular function and the modulation of health and disease. Front Cardiovasc Med 2017; 4: 1-11

[14] Brocq ML, Leslie SJ, Milliken P, Megson IL. Endothelial dysfunction: from molecular mechanisms to measurement, clinical implications and therapeutic opportunities. Antioxid Redox Signal 2008; 10: 1631-1674

[15] Božunović J, Živković S, Gašić U, Glamočlija J, Ćirić A, Matekalo D, Šiler B, Soković M, Tešić Ž, Mišić D. In vitro and in vivo transformations of Centaurium erythraea secoiridoid glucosides alternate their antioxidant and antimicrobial capacity. Ind Crops Prod 2018; 111: 705-721

[16] Ghiadoni L, Taddei S, Virdis A. Hypertension and endothelial dysfunction: therapeutic approach. Curr Vasc Pharmacol 2011; 10: 43-60

[17] Goszcz K, Deakin SJ, Duthie GG, Stewart D, Leslie SJ, Megson IL. Antioxidants in cardiovascular therapy: panacea or false hope? Front Cardiovasc Med 2015; 2: 1-22 
[18] Vanhoutte PM, Shimokawa H, Feletou M, Tang EHC. Endothelial dysfunction and vascular disease - a 30th anniversary update. Acta Physiol (Oxf) 2017; 219: 22-96

[19] Mombouli JV, Vanhoutte PM. Endothelium-derived hyperpolarizing factor(s): updating the unknown. Trends Pharmacol Sci 1997; 18: 252-256

[20] Nelson MT, Quayle JM. Physiological roles and properties of potassium channels in arterial smooth muscle. Am J Physiol 1995; 4: C799-C822

[21] Furchgott RF. The role of endothelium in the responses of vascular smooth muscle to drugs. Annu Rev Pharmacol Toxicol 1984; 24: 175197

[22] McAnulty RJ. Fibroblasts and myofibroblasts: their source, function and role in disease. Int J Biochem Cell Biol 2007; 39: 666-671

[23] Brecher P. Angiotensin II and cardiac fibrosis. Trends Cardiovasc Med 1996; 6: 193-198

[24] Kim S, Iwao H. Molecular and cellular mechanisms of angiotensin II-mediated cardiovascular and renal diseases. Pharmacol Rev 2000; 52: $11-$ 34

[25] Porter KE, Turner NA. Cardiac fibroblasts: at the heart of myocardial remodeling. Pharmacol Ther 2009; 123: 255-278

[26] van Eickels M, Vetter H, Grohe C. Angiotensin-converting enzyme (ACE) inhibition attenuates insulin-like growth factor-I (IGF-I) induced cardiac fibroblast proliferation. Br J Pharmacol 2000; 131: 1592-1596

[27] Sadoshima J, Izumo S. Molecular characterization of angiotensin II-induced hypertrophy of cardiac myocytes and hyperplasia of cardiac fibroblasts. Critical role of the AT1 receptor subtype. Circ Res 1993; 73: 413423

[28] Kachmar MR, Oliveira AP, Valentão P, Gil-Izquierdo A, Domínguez-Perles R, Ouahbi A, Badaoui KE, Andrade PB, Ferreres F. HPLC-DAD-ESI/MSn phenolic profile and in vitro biological potential of Centaurium erythraea Rafn aqueous extract. Food Chem 2019; 278: 424-433

[29] Sanchez M, Lodi F, Vera R, Villar IC, Cogolludo A, Jimenez R, Moreno L, Romero M, Tamargo J, Perez-Vizcaino F, Duarte J. Quercetin and isorhamnetin prevent endothelial dysfunction, superoxide production, and overexpression of p47phox induced by angiotensin II in rat aorta. J Nutr 2007; 137: 910-915

[30] Penso ], Cordeiro K, da Cunha CR, da Silva Castro PF, Martins DR, Lião LM, Rocha ML, de Oliveira V. Vasorelaxant activity of 7- $\beta$-O-glycosides biosynthesized from flavonoids. Eur ] Pharmacol 2014; 733: 75-80

[31] Hügel HM, Jackson N, May B, Zhang AL, Xue CC. Polyphenol protection and treatment of hypertension. Phytomedicine 2016; 23: 220-231

[32] Peluso MR. Flavonoids attenuate cardiovascular disease, inhibit phosphodiesterase, and modulate lipid homeostasis in adipose tissue and liver. Exp Biol Med 2006; 231: 1287-1299
[33] Gasparotto Junior A, Dos Reis Piornedo R, Assreuy ], Da Silva-Santos JE. Nitric oxide and Kir6. 1 potassium channel mediate isoquercitrin-induced endothelium-dependent and independent vasodilation in the mesenteric arterial bed of rats. Eur ] Pharmacol 2016; 788: 328-334

[34] Mahobiya A, Singh TU, Rungsung S, Kumar T, Chandrasekaran G, Parida $S$, Kumar D. Kaempferol-induces vasorelaxation via endothelium-independent pathways in rat isolated pulmonary artery. Pharmacol Rep 2018; 70: 863-874

[35] Xiao H, Lu X, Chen X, Sun Z. Protective effects of kaempferol against endothelial damage by an improvement in nitric oxide production and a decrease in asymmetric dimethylarginine level. Eur J Pharmacol 2009; 616: 213-222

[36] Teixeira J, Gaspar A, Garrido EM, Garrido J, Borges F. Hydroxycinnamic acid antioxidants: an electrochemical overview. Biomed Res Int 2013; 2013: 251754. doi:10.1155/2013/251754

[37] Mudnic I, Modun D, Rastija V, Vukovic J, Brizic I, Katalinic V, Kozina B, Medic-Saric M, Boban M. Antioxidative and vasodilatory effects of phenolic acids in wine. Food Chem 2010; 119: 1205-1210

[38] Brand-Williams W, Cuvelier ME, Berset C. Use of a free radical method to evaluate antioxidant activity. Lebensm Wiss Technol 1995; 28: 25-30

[39] Mcgregor DD. The effect of sympathetic nerve stimulation of vasoconstrictor responses in perfused mesenteric blood vessels of the rat. J Physiol 1965; 177: 21-30

[40] Bencheikh R, Féray JC, Alaoui A, Crozatier B. Thromboxane A2 in vasomotor effects of phenylephrine, acetylcholine, and bradykinin in rat mesenteric bed. J Cardiovasc Pharmacol 2002; 40: 255-264

[41] Adeagbo ASO, Tabrizchi R, Triggle CR. The effects of perfusion rate and NG-nitro-L-arginine methyl ester on cirazoline- and $\mathrm{KCl}$-induced responses in the perfused mesenteric arterial bed of rats. $\mathrm{Br}$ J Pharmacol 1994; 111: 13-20

[42] Chda A, El Kabbaoui M, Baba BF, Mahfoud A, El Abida K, Bousfiha A, Al Houari G, Abdelali T, Kerbal A, Ben Cheikh R. Endothelium-independent vasorelaxant effect of synthestized 2-hydroxymethylchromone on rat mesenteric arterial bed. Curr Bioact Compd 2018; 14: 289-298

[43] Green L, Wagner D, Glogowski J, Skipper P, Wishnok JS, Tannenbaum S. Analysis of nitrate, nitrite, and $\left[{ }^{15} \mathrm{~N}\right]$ nitrate in biological fluids. Anal Biochem 1982; 126: 131-138

[44] Certal M, Vinhas A, Pinheiro AR, Ferreirinha F, Barros-Barbosa AR, Silva I, Costa MA, Correia-de-Sá P. Calcium signaling and the novel antiproliferative effect of the UTP-sensitive P2Y11 receptor in rat cardiac myofibroblasts. Cell Calcium 2015; 58: 518-533

[45] Denizot F, Lang R. Rapid colorimetric assay for cell growth and survival. Modifications to the tetrazolium dye procedure giving improved sensitivity and reliability. J Immunol Methods 1986; 89: 271-277 